\title{
Functional Development of Engineered Skeletal Muscle from Adult and Neonatal Rats
}

\author{
PAUL E. KOSNIK, Ph.D., JOHN A. FAULKNER, Ph.D., \\ and ROBERT G. DENNIS, Ph.D.
}

\begin{abstract}
A myooid is a three-dimensional skeletal muscle construct cultured from mammalian myoblasts and fibroblasts. The purpose was to compare over several weeks in culture the morphology, excitability, and contractility of myooids developed from neonatal and adult rat cells. The hypotheses tested were as follows: (1) baseline forces of myooids correlate with the cross-sectional area (CSA) of the myooids composed of fibroblasts, and (2) peak isometric tetanic forces normalized by total CSA (specific $P_{0}$ ) of neonatal and adult rat myooids are not different. Electrical field stimulation was used to measure the excitability and peak tetanic forces. The proportion of the CSA composed of fibroblasts was greater for neonatal $(40 \%)$ than adult $(\mathbf{1 7 \%})$ myooids. For all myooids the baseline passive force normalized by fibroblast CSA $($ mean $=5.5 \mathrm{kPa})$ correlated with the fibroblast $\mathrm{CSA}\left(\boldsymbol{r}^{2}=0.74\right)$. A two-element cylindrical model was analyzed to determine the contributions of fibroblasts and myotubes to the baseline force. At each measurement period, the specific $P_{0}$ of the adult myooids was greater than that of the neonatal myooids. The specific $P_{0}$ of the adult myooids was $\sim 1 \%$ of the control value for adult muscles and did not change with time in culture, while that of neonatal myooids increased.
\end{abstract}

\section{INTRODUCTION}

D ESPITE OBSERVATIONS OF spontaneous contractions of cultured mammalian skeletal muscle cells as early as $1915,{ }^{1,2}$ forces generated by three-dimensional skeletal muscle constructs tissue engineered in vitro have been measured only recently.,4 The first measurements of contractility were made on avian skeletal muscle constructs, termed organoids. ${ }^{3}$ More recently, mammalian skeletal muscle constructs, termed myooids, were engineered in vitro and functional measurements made of excitability and isometric contractile properties. ${ }^{4}$ Although these measurements allowed for direct comparison of the contractile properties of myooids with control skeletal muscle, the data were collected at a single time point in culture of $\sim 30$ days. Consequently, any changes in the contractile properties of the myooids associated with the length of time in culture were not considered.

Skeletal muscle constructs developed from coculture of myoblasts and fibroblasts have some percentage of their cross-section that consists primarily of an extracellular matrix (ECM) and fibroblasts. ${ }^{3-6}$ When organoids $s^{3}$ and myooids ${ }^{4}$ are viewed in cross-section, fibroblasts form an annulus that surrounds a core of

Department of Biomedical Engineering and Institute of Gerontology, University of Michigan, Ann Arbor, Michigan. 
myotubes. Organoids and myooids display significant baseline passive forces that may result from this population of fibroblasts. ${ }^{7}$ The forces generated by fibroblasts have been studied both in vitro and in vivo. When normalized by fibroblast cross-sectional area (CSA), avian fibroblasts in a collagen gel ${ }^{8}$ developed stresses of $4.5 \mathrm{kPa}$, sheets of avian fibroblasts in their own $\mathrm{ECM}^{9}$ developed stresses of $3.4 \mathrm{kPa}$, and the mean wound closure stress generated by fibroblasts in rabbits ${ }^{10}$ was $3.2 \mathrm{kPa}$. For clarity and in keeping with the conventions employed in the current body of literature, the active normalized forces, generated as a result of electrical stimulation of muscle tissue, are reported in units of $\mathrm{kN} / \mathrm{m}^{2}$, whereas the passive forces from any source within the tissue other than actively contracting muscle are reported in units of $\mathrm{kPa}$.

The purpose of this study was to compare over several weeks in culture the morphology, excitability, and contractility of myooids developed from neonatal and adult rat cells. The hypotheses tested were as follows: (1) baseline forces of myooids correlate with the CSA of the myooids composed of fibroblasts, and (2) peak isometric tetanic force normalized by total CSA (specific Po) of neonatal and adult rat myooids are not different.

\section{MATERIALS AND METHODS}

Previously described methods were used to engineer myooids and measure their excitability and contractility. ${ }^{4}$ Satellite cells and fibroblasts were harvested from a single litter of 13 neonatal Sprague Dawley rats that were born and shipped the same day (Charles River, Wilmington, MA), and one adult Sprague Dawley rat (Charles River). Rats were cared for under University of Michigan guidelines governing the use of animals for laboratory research. Between 4 and 31 days after myooid formation, morphology, excitability, and contractility were measured on 26 neonatal rat myooids and 14 adult rat myooids.

\section{Culture plates and media}

Culture dishes were prepared with laminin-coated suture anchors pinned in place on a SYLGARD (type 184; Dow Chemical Corporation, Midland, MI) substrate. ${ }^{4}$ The culture media were growth medium (GM), consisting of $400 \mathrm{~mL}$ of HAM F-12 nutrient mixture (GibcoBRL \#11765-054) with $100 \mathrm{~mL}$ of fetal bovine serum (FBS, GibcoBRL \#10437-036) and 100 units/mL of Penicillin G (SIGMA \#P-3414), and differentiation medium (DM), consisting of $465 \mathrm{~mL}$ of DMEM (GibcoBRL \#11995-065) with $35 \mathrm{~mL}$ of horse serum (HS, GibcoBRL \#16050-114) and 100 units/mL Penicillin G. ${ }^{4}$ Mincing solution ${ }^{11}$ consisted of $8 \mathrm{mM}$ $\mathrm{NaH}_{2} \mathrm{PO}_{4}, 22.6 \mathrm{mM} \mathrm{NaHCO} 3,116 \mathrm{mM} \mathrm{NaCl}, 5.3 \mathrm{mM} \mathrm{KCl}, 5.6 \mathrm{mM}$ glucose, $\mathrm{pH} \mathrm{7.4,} \mathrm{stored} \mathrm{at} 4{ }^{\circ} \mathrm{C}$. In addition, a preincubation medium (PIM) and a dissociation solution of dispase (GibcoBRL \#17105-041) and type IV collagenase (Worthington \#CLS 4, Lakewood, NJ) in DMEM were prepared. ${ }^{4}$

\section{Cell harvesting and culture}

To harvest cells from neonatal rat tissue, pups from a litter of neonatal rats were placed on ice for approximately $45 \mathrm{~min}$ as a general anesthesia. Neonatal pups were then dipped in a solution of $70 \%$ ethanol in order to decrease the chances of later tissue culture contamination. All procedures thereafter were carried out using aseptic procedures in a class IIA/B3 biological safety cabinet. After removal of the paws and skin, the muscle tissue from all four limbs of each neonate was surgically removed and placed in a 60-mmdiameter culture dish with $8 \mathrm{~mL}$ of mincing solution. The harvested tissue was transferred to a 50-mL conical tube containing $12 \mathrm{~mL}$ of dissociating solution and stirred with a magnetic bar for $1 \mathrm{~h}$. The dissociated tissue was then centrifuged at $1,200 G$ for $15 \mathrm{~min}$. After the removal of the supernatant, the tissue was resuspended in $20 \mathrm{~mL}$ of F12 Nutrient Mixture with $20 \mathrm{mg}$ of collagenase type IV and incubated at $37^{\circ} \mathrm{C}$ for $1 \mathrm{~h}$. The tube was again centrifuged at $1,200 \mathrm{G}$ for $15 \mathrm{~min}$, after which the supernatant was removed and the cells were resuspended in GM. Cell density was determined using a hemacytometer; cells were plated at $1 \times 10^{7}$ cells/plate. Harvested cells from neonatal rats were not preincubated, and were plated on previously prepared culture dishes with laminin-coated substrate and artificial tendons in place.

Cells were harvested from the soleus muscles of one adult (5-6-month-old) Sprague-Dawley rat, after being subjected to $48 \mathrm{~h}$ of preincubation in PIM at $37^{\circ} \mathrm{C}$ and $5 \% \mathrm{CO}_{2} \cdot{ }^{4}$ After preincubation, the muscle 
strips were dissociated enzymatically to release myoblasts and fibroblasts for primary culture. Due to the presence of tissue debris after dissociation, myogenic precursor cells could not be counted accurately enough to determine the plating density. Cell plating density was recorded as the amount of tissue mass dissociated for the formation of each myooid, $\sim 10 \mathrm{mg} / \mathrm{myooid}$. After plating, the dishes were placed in culture at $37^{\circ} \mathrm{C}$ and $5 \% \mathrm{CO}_{2}$, and were not handled for $48 \mathrm{~h}$.

For both neonatal and adult rat cell cultures, the GM was replaced at $48 \mathrm{~h}$ after cell plating and three times per week thereafter until the cells became confluent, which required $\sim 4$ days for neonatal cultures and $\sim 8$ days for adult cultures. After reaching confluence, the cells were fed three times per week with DM. Within a few days of switching to DM, the confluent cells typically fused to form multinucleated myotubes that began to spontaneously contract. After 2-3 weeks in culture, the monolayer detached from the SYLGARD substrate and the myooids formed. Two myooids from each group underwent in vitro contractile function tests at day 1 after myooid formation, and four myooids from each group were evaluated for contractile function on days $4,8,15$, and 31 after myooid formation. Comparisons at specific times between neonatal and adult rat myooids were made from the time of myooid formation because physical remodeling of the myotubes and fibroblasts in the myooids occurred from the time of myooid formation, and these phenomena presumably have greater impact on the excitability and contractile function of the myooids than does the time in culture. Comparisons made from the time of myooid formation do present the possibility that differences between the two groups in time from cell plating until myooid formation may result in differences in cell phenotype, thus confounding comparisons between groups made from time of myooid formation.

\section{Measurement of excitability and contractility}

Myooids were maintained at a length of $12 \mathrm{~mm}$ between suture anchors, referred to as the culture length $\left(\mathrm{L}_{\mathrm{c}}\right)$, for measurement of contractile properties. Myooid diameter was measured using a calibrated eyepiece reticule with a resolution of $5 \mu \mathrm{m}$. The total CSA of each myooid was calculated on the basis of the measured diameter, with the assumption that each myooid approximates a cylinder. ${ }^{4}$ Forces were measured by attaching one of the suture anchors to a force transducer, and electrical stimulation was applied via parallel platinum wire electrodes using the experimental apparatus reported in an earlier publication. ${ }^{4}$

Baseline force $\left(\mathrm{P}_{\mathrm{b}}\right)$, the force generated by the myooid while not electrically excited to generate active force, was measured. Specific $\mathrm{P}_{\mathrm{b}}$ was calculated by dividing $\mathrm{P}_{\mathrm{b}}$ by the total CSA of fibroblasts. Peak twitch force $\left(\mathrm{P}_{t}\right)$ was determined by activating the myooid with a single 70-volt, 4-msec wide pulse between the parallel platinum wire electrodes. Peak isometric tetanic force $\left(\mathrm{P}_{\mathrm{o}}\right)$ was determined by stimulating the myooid at 40 volts and $40 \mathrm{~Hz}$ with 1.20 -msec wide pulses for a train duration of $2 \mathrm{sec}$. Specific $\mathrm{P}_{\mathrm{o}}$ was calculated by dividing $\mathrm{P}_{\mathrm{o}}$ by the total CSA of the myooid.

Excitability was assessed by measures of rheobase and chronaxie. ${ }^{4}$ For a single stimulus pulse, rheobase is a measure of the minimum stimulus amplitude required to elicit an action potential in a particular preparation, and chronaxie is a measure of the stimulus duration. These measures have been classically applied to the depolarization threshold of individual cells or specific regions of a cell membrane. For a tissue construct with many cells, the classical definitions of rheobase and chronaxie, although still useful, must be modified. For muscle tissue, the result of the application of a single stimulus pulse is a twitch. In preliminary experiments, a large matrix of stimulus pulse amplitudes and widths were applied to the myooids to determine supramaximal pulse amplitudes and pulse durations. ${ }^{4}$ A pulse width of 4-6 msec was always sufficient to elicit maximal twitch force, and the relationship between stimulus amplitude and twitch force was sigmoidal with maximum slope in the region of ${ }^{1} /{ }_{2} \mathrm{P}_{\mathrm{t}}$. The rheobase $\left(\mathrm{R}_{50}\right)$ was measured by fixing the pulse duration at $6 \mathrm{~ms}$, and then determining the voltage field strength to elicit a twitch of ${ }^{1} /{ }_{2} \mathrm{P}_{\mathrm{t}}$. The voltage was divided by the electrode separation $(\mathrm{mm})$ and $\mathrm{R}_{50}$ was reported as electric field strength in units of $\mathrm{V} / \mathrm{mm}$. Chronaxie $\left(\mathrm{C}_{50}\right)$ was determined by fixing the field strength to twice the rheobase voltage, and adjusting the pulse duration to again elicit twitch forces of ${ }_{1} / \mathrm{P}_{\mathrm{t}}$. Measurements of $\mathrm{R}_{50}$ and $\mathrm{C}_{50}$ are dependant on the specific preparation and electrode configuration, but relative changes in tissue excitability, with time or other interventions, can be detected readily. As with $\mathrm{R}_{50}$, higher values of $\mathrm{C}_{50}$ indicate lower excitability. 


\section{Morphology}

After measuring the excitability and contractility, each myooid was pinned at $\mathrm{L}_{\mathrm{c}}$ and fixed for $4 \mathrm{~h}$ at $4{ }^{\circ} \mathrm{C}$ in Karnovsky's solution (0.1 M sodium cacodylate buffer with 3\% formaldehyde and 3\% glutaraldehyde at $\mathrm{pH}$ 7.4). Myooids were rinsed three times (30 min, $30 \mathrm{~min}$, and $4 \mathrm{~h}$ ) with cacodylate buffer ( $\mathrm{pH} 7.4$ ) with $7.5 \%$ sucrose. Myooids were postfixed in $1 \%$ osmium tetroxide for $2 \mathrm{~h}$ at room temperature, dehydrated in graded concentration of ethanol and propylene oxide, and embedded in EPON (Eponate 12 resin, Ted Pella Inc., Redding, CA) for light microscopy. Cross-sections were cut $600 \mathrm{~nm}$ thick for light microscopy and stained with $1 \%$ Toluidine blue.

The total CSA of myooids was comprised chiefly of two cell types, myotubes and fibroblasts, in addition to extracellular matrix (ECM). ${ }^{4}$ The passive and active forces generated by myooids were normalized by the appropriate CSA to permit comparisons of contractility of myooids with different diameters. To determine the relative contributions of myotubes and fibroblasts to passive and active forces, each myooid was sectioned and the cross sectional areas occupied by each type of cell were measured. Myotube CSA was determined by measuring the total CSA and the fibroblast CSA using a BIOQUANT (R \& M Biometrics, Inc.) system by estimating the boundary between the two cell types based on cell morphology, and taking the difference between the two areas.

During longitudinal studies of myooids in culture, the fibroblast and myotube CSA cannot be determined without removing a representative population of myooids, sectioning and staining them. Therefore, for comparison with longitudinal data, myooid forces were normalized by both total CSA, as well as by either fibroblast or myotube CSA, depending on whether the forces were passive or active. The diameter, and thus the total CSA, of myooids decreases with time in culture. ${ }^{4}$ In addition, the internal structure of each myooid remodels rapidly, and there is currently no evidence that the relative area of myotubes and fibroblasts within a myooid remains constant over time. Two-tailed Student's $t$ tests (INSTAT) were performed to compare adult and neonatal rat myooid contractile properties at specific times after myooid formation.

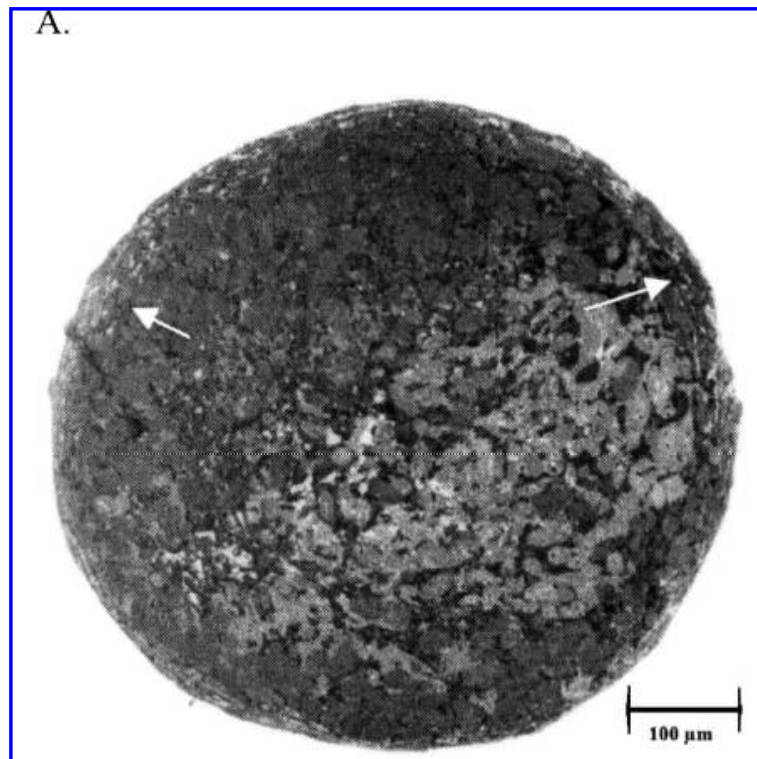

B.

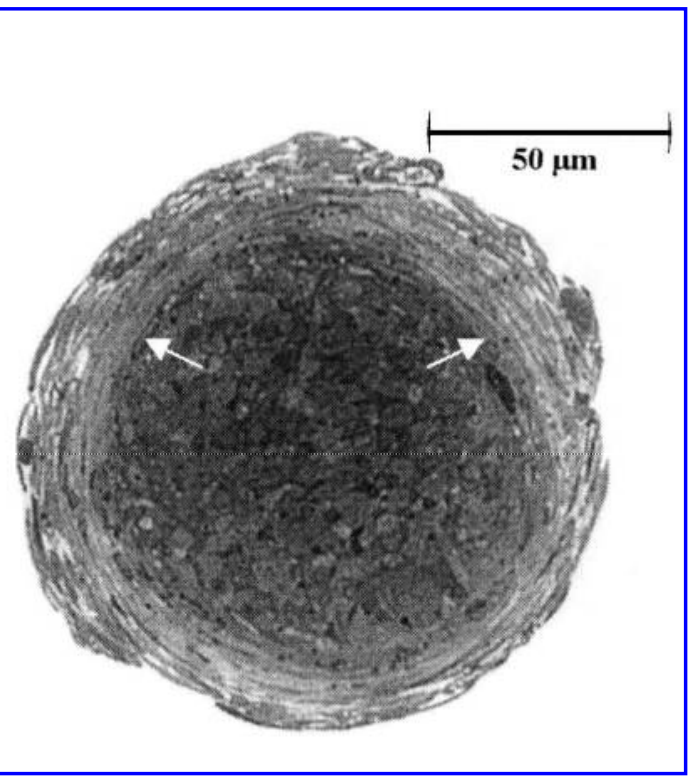

FIG. 1. Cross-sections of adult (A) and neonatal (B) myooids. The boundary between the interior core of myotubes and annulus of fibroblasts and ECM is indicated by arrows. (A). Typical adult rat myooid cross-section at day 11 after myooid formation stained with $1 \%$ Toluidine blue. (B). Typical neonatal rat myooid cross-section at day 25 after myooid formation stained with $1 \%$ Toluidine blue. Note the difference in the relative areas occupied by fibroblasts and their associated ECM. 


\section{RESULTS}

\section{Myooid formation}

Myoblasts and fibroblasts proliferated to form a confluent monolayer of cells. Myocytes subsequently fused to form randomly oriented multinucleated myotubes that began to contract spontaneously. The spontaneous contractions accelerated the delamination of the monolayer from the SYLGARD substrate, but the monolayer remained attached to the suture anchors. Subsequent remodeling of the monolayer resulted in a cylindrical engineered tissue. Myooids were held under tension above the substrate between the two artificial tendons by the development of $\mathrm{P}_{\mathrm{b}}{ }^{4}$ Neonatal rat myooids first formed after $11 \pm 5$ days in culture and adult rat myooids first formed after $21 \pm 3$ days in culture. Neonatal rat myooids survived for 39 days after formation, when the last group was evaluated for contractile properties. Due to contamination of some of the plates, a reduced number of adult rat myooids was available, limiting the time course for the measurements on the adult rat myooids to 18 days.

\section{Morphology}

Fibroblasts were organized around the periphery of the myooids, but the interior of the myooids were composed predominately of myotubes (Fig. 1). The fibroblast CSA of neonatal rat myooids was $40 \%$ of the total CSA, larger $(p<0.01)$ than the $17 \%$ observed for adult rat myooids (Fig. 1). Once myooids formed, their diameter generally decreased with time in culture (Fig. 2). An exception to this trend occurred for neonatal rat myooids between days 25 and 32 after myooid formation (Fig. 2). At this time, myoblasts and fibroblasts from neonatal rats that had remained on the SYLGARD substrate after initial myooid formation continued to proliferate in culture, formed a new monolayer, delaminated, and were incorporated into the existing myooid. The newly incorporated tissue resulted in a twofold increase in neonatal rat myooid diameter. By day 32 after myooid formation, the new tissue could still be identified from the initial myooid tissue since the new tissue had not yet been fully incorporated into the original myooid (Fig. 3).

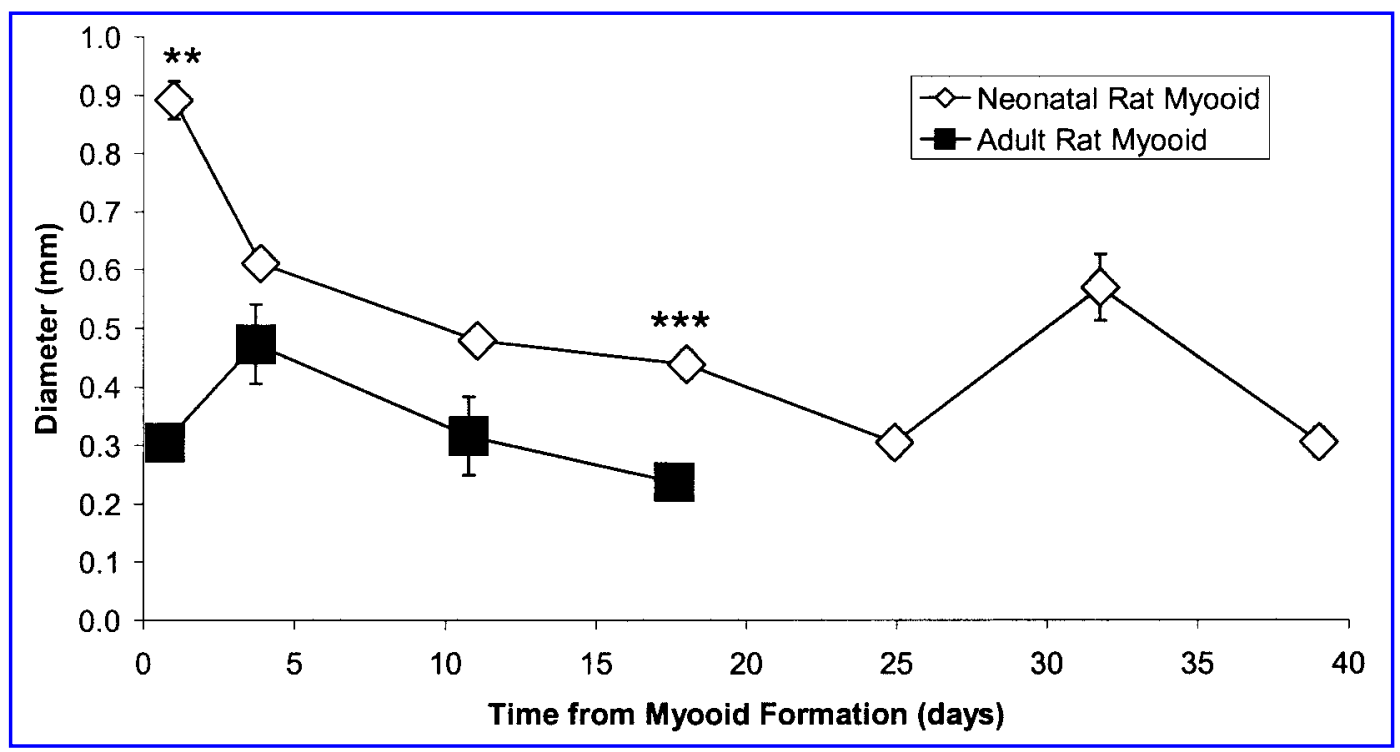

FIG. 2. Diameter of neonatal and adult rat myooids plotted against time after myooid formation. A secondary delamination occurred in several neonatal rat culture plates and the resultant sheet of tissue was incorporated into the myooids before the diameter measurement on day 32 after myooid formation. The first datum of each sequence is the average of two myooid diameters. All other data points for both groups are the mean \pm SEM for four myooid diameters. Statistically significant differences between the groups: $* p<0.05, * * p<0.01$, and $* * * p<0.001$. 


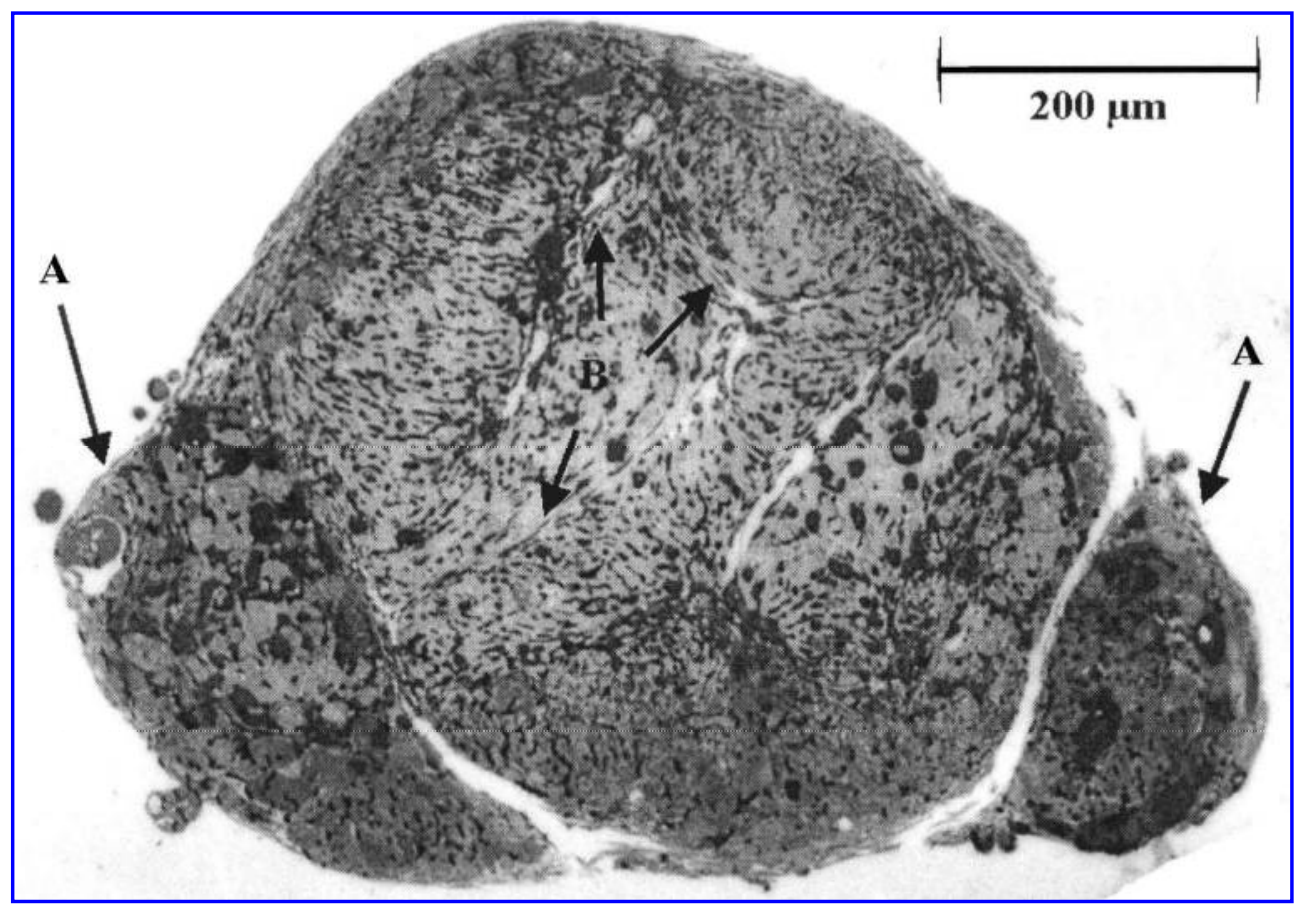

FIG. 3. Myooid developed from neonatal rat myogenic precursor cells stained with $1 \%$ Toluidine blue. A secondary delamination of the cultured cells occurred, and the resultant tissue was incorporated into the myooid. The new tissue has not been completely integrated into the structure (A). Note the separation of the myooid into fascicles (B). Day 32 after myooid formation.

\section{Passive baseline stresses}

All myooids had a significant $\mathrm{P}_{\mathrm{b}}$ at $\mathrm{L}_{\mathrm{c}}$. For 20 neonatal and adult rat myooids, a plot of the myooid CSA composed of fibroblasts with the corresponding $\mathrm{P}_{\mathrm{b}}$ for each myooid reveals a strong correlation between $\mathrm{P}_{\mathrm{b}}$ and fibroblast CSA (Fig. $\left.4 ; F<0.001\right)$. The mean neonatal rat myooid $\mathrm{P}_{\mathrm{b}}\left(2.55 \times 10^{-7} \pm 2.82 \times 10^{-8}\right.$ $\mathrm{kN})$ was greater $(p<0.001)$ than that for adult rat myooids $\left(1.03 \times 10^{-7}+1.20 \times 10^{-8} \mathrm{kN}\right)$, although when normalized by CSA to determine the specific $\mathrm{P}_{\mathrm{b}}$, the means were not different. The linear regression included in Figure 4 indicates that the baseline force correlates strongly with the CSA of fibroblasts $\left(\mathrm{CSA}_{\mathrm{f}}\right)$ within each myooid. The units of measure on each axis of Figure 4 were selected such that the resulting slope of the linear regression had units of $\mathrm{kPa}$ to estimate the passive stress resulting from the fibroblasts and their associated ECM. With the assumption that $\mathrm{P}_{\mathrm{b}}$ results only from fibroblasts and their associated ECM, the tensile stress of the fibroblasts along the longitudinal axis of the myooid $\left(\sigma_{\mathrm{f}}\right)$ was calculated to be $\sim 5.5 \mathrm{kPa}$.

To estimate the contribution of the longitudinal passive tensile stress of the myotubes to $\mathrm{P}_{\mathrm{b}}$, we constructed a two-element model in which a cylinder of myotubes is surrounded by a hollow tube of fibroblasts of uniform wall thickness. In this model, the measured value of $\mathrm{P}_{\mathrm{b}}$ is the sum of passive forces generated both by the annulus of fibroblasts and their associated ECM as well as the core of myotubes. The total force generated by each of these components of the myooid can be expressed as:

$$
\mathrm{P}_{\mathrm{b}}=\sigma_{\mathrm{f}} \mathrm{CSA}_{\mathrm{f}}+\sigma_{\mathrm{m}} \mathrm{CSA}_{\mathrm{m}}+\mathrm{P}_{\text {offset }}
$$

In Equation 1, $\mathrm{CSA}_{\mathrm{f}}$ and $\mathrm{CSA}_{\mathrm{m}}$ represent the measured values of the cross-sectional areas of the fibroblasts and the myotubes in each myooid, respectively, $\sigma_{\mathrm{f}}$ and $\sigma_{\mathrm{m}}$ are the longitudinal component of the passive baseline stress within the fibroblasts and the myotubes, and $\mathrm{P}_{\text {offset }}$ is the remaining baseline force mea- 
surement, which would include any uncorrected force transducer offset, as well as higher-order terms. This characteristic equation applies to each myooid in the data set of 20 individual myooids, and taken as a whole constitutes a system of 20 simultaneous linear equations. The coefficients $\sigma_{\mathrm{m}}, \sigma_{\mathrm{f}}$, and $\mathrm{P}_{\text {offset }}$ are vastly overdetermined by the 20 simultaneous, and typically inconsistent, system of equations. The standard approach is to solve the multiple regression problem to find the least squares fit of the data by constructing the equations in matrix form. The method of calculating the coefficients $\sigma_{\mathrm{m}}, \sigma_{\mathrm{f}}$, and $\mathrm{P}_{\mathrm{offset}}$ is presented in the Appendix. The resulting values for the coefficients were: $\sigma_{\mathrm{f}}=5.9 \mathrm{kPa}, \sigma_{\mathrm{m}}=-0.18 \mathrm{kPa}$, and $\mathrm{P}_{\text {offset }}=$ $7.7 \times 10^{-8} \mathrm{kN}$. The value of $\mathrm{P}_{\text {offset }}$ includes forces generated by sources other than the myotubes or fibroblasts (or their associated ECM), as well as systematic experimental error, such as force transducer offset. In keeping with the convention of the literature, passive normalized forces including those arising within muscle are reported in units of $\mathrm{kPa}$, whereas the normalized active forces are reported as $\mathrm{kN} / \mathrm{m}^{2}$, even though the dimensions are identical.

The mechanical stress generated by sheets of fibroblasts in culture is biaxial. ${ }^{8,9}$ Therefore, in addition to the longitudinal stress generated by the fibroblasts in the myooid, the annulus of fibroblasts around the periphery exerts a tangential stress on the cylindrical surface of the myooid. This is equivalent to the welldescribed hoop stress in the walls of cylindrical pressure vessels, and allows the calculation of the internal pressure within the myooid that results from the tangential stress from fibroblasts at the periphery. Assuming that the biaxial passive stress generated by the fibroblasts was uniform, i.e., the tangential stress was the same as the longitudinal stress $\left(\sigma_{\mathrm{ft}}=\sigma_{\mathrm{f}}\right)$, we can employ the standard equation for pressure vessels of any wall thickness ${ }^{16}$ to calculate the internal pressure within a myooid:

$$
\sigma_{\mathrm{ft}}=\mathrm{pd}_{\mathrm{i}} / 2 \mathrm{t}
$$

thus,

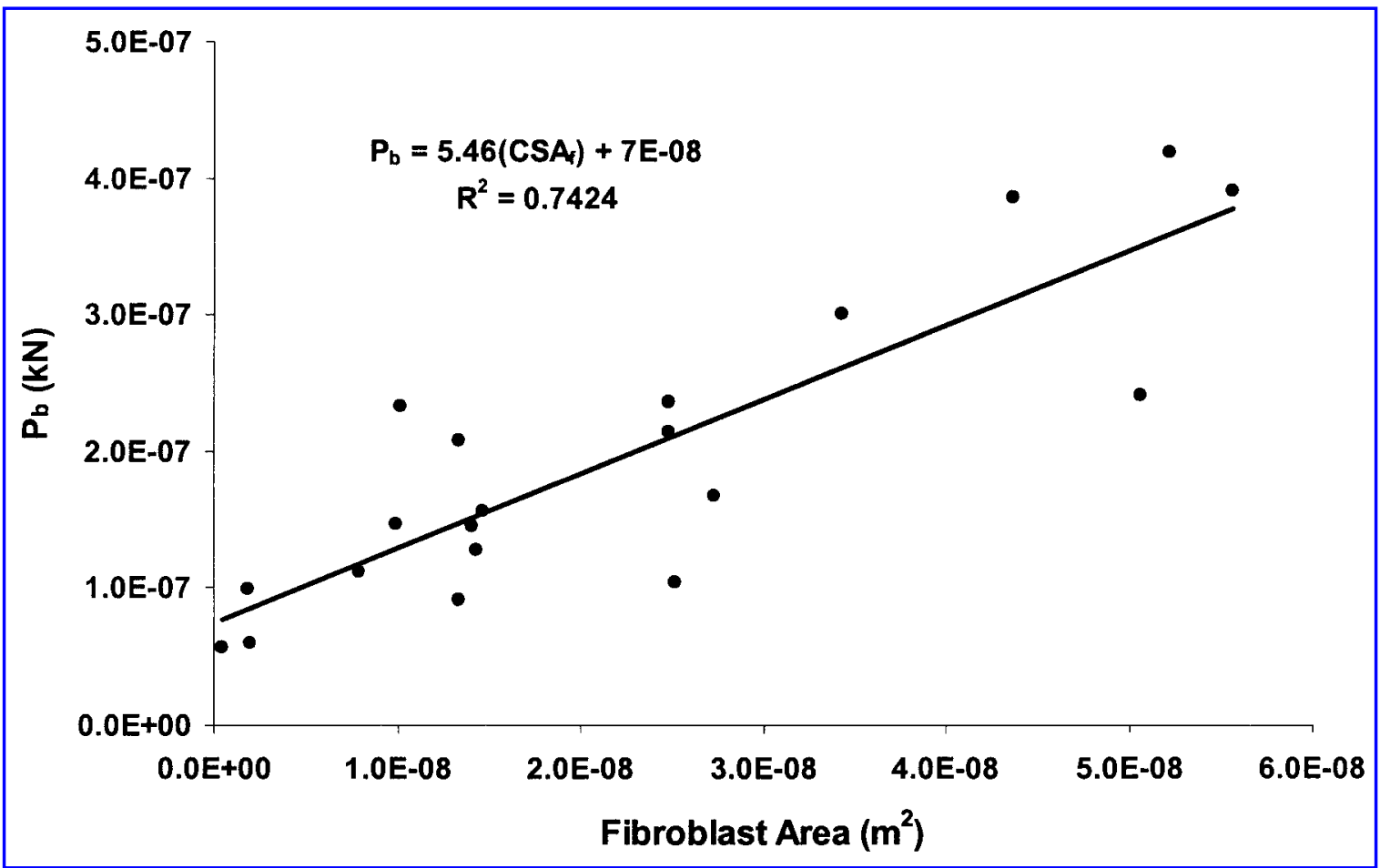

FIG. 4. $\mathrm{P}_{\mathrm{b}}$ as a function of the CSA of fibroblasts in each myooid. A significant $\left(r^{2}=0.74 ; F<0.001\right)$ correlation exists between resting baseline force of myooids and the total fibroblast CSA $\left(\mathrm{CSA}_{\mathrm{f}}\right)$ of the myooid. Data are for individual adult and neonatal rat myooids. The slope of the linear regression line is the specific $\mathrm{P}_{\mathrm{b}}$ of fibroblasts in myooids, $\sim 5.5 \mathrm{kPa}$. 


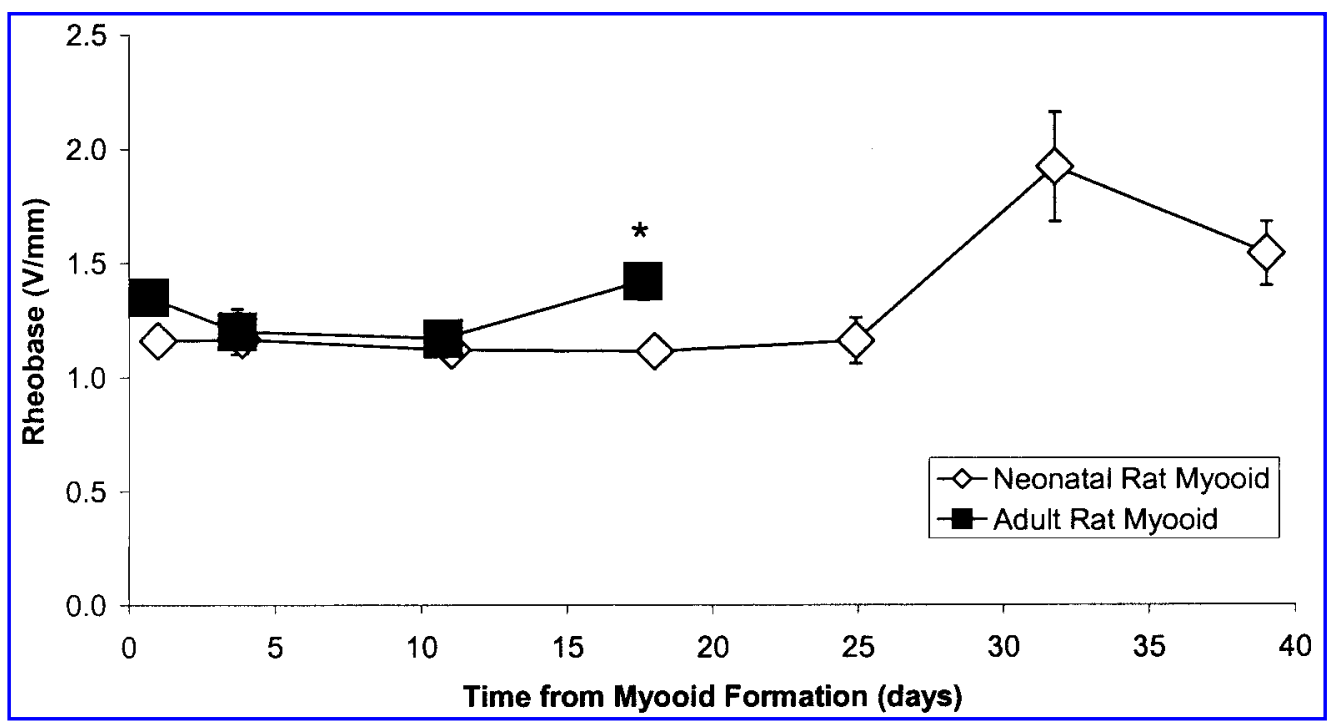

FIG. 5. Rheobase of adult and neonatal rat myooids. The first datum of each sequence is the average of two myooid rheobase values. All other data points for both groups are the mean \pm SEM for four myooid rheobase values. Statistically significant differences between the groups: $* p<0.05, * * p<0.01$, and $* * * p<0.001$.

$$
\mathrm{p}=\sigma_{\mathrm{ft}} 2 \mathrm{t} / \mathrm{d}_{\mathrm{i}}
$$

Using the calculated value of $\sigma_{\mathrm{f}}$, from the solved vector of coefficients from Equation 4 (Appendix) to estimate the tangential stress generated by the fibroblasts $\left(\sigma_{\mathrm{ft}}\right)$, and the measured myotube core diameter $\left(\mathrm{d}_{\mathrm{i}}\right)$ and the fibroblast wall thickness $(\mathrm{t})$, we calculate that the average pressure ( $\mathrm{p}$ ) on the core of myotubes for all myooids in this study is $\sim 1 \mathrm{kPa}$. From Equation 2, it is clear that thicker fibroblast layers (t), as well as smaller muscle core diameters $\left(\mathrm{d}_{\mathrm{i}}\right)$, will result in increased internal pressure $(\mathrm{p})$ within the myooid. Myooids with greater baseline forces have been shown to have reduced lifespan in culture. ${ }^{17}$ The explana-

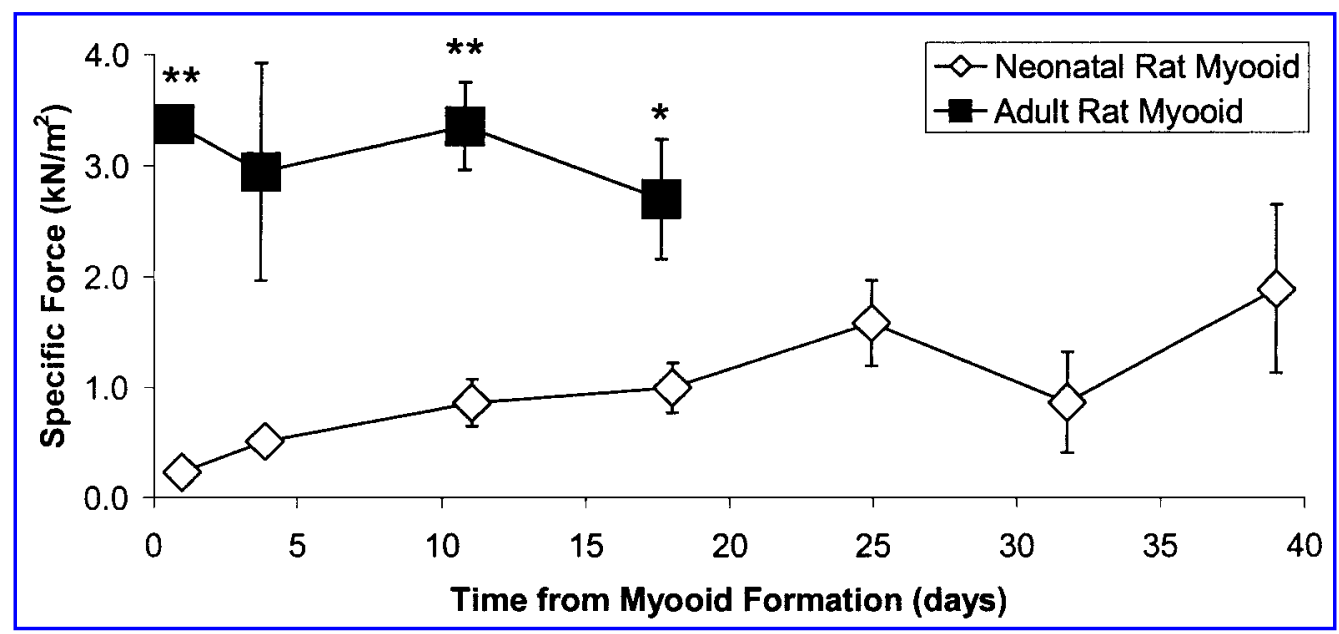

FIG. 6. Specific $\mathrm{P}_{\mathrm{o}}$ for adult and neonatal rat myooids. The total myooid CSA was used to calculate specific force. The first datum of each sequence is the average of two myooid specific forces. All other data points for both groups are the mean \pm SEM for four myooid specific forces. Statistically significant differences between the groups: $* p<$ $0.05, * * p<0.01$, and $* * * p<0.001$. 
tion for this phenomenon may be that the pressure exerted by the fibroblasts results in accelerated degeneration of the myotubes in the myooids, by a mechanism similar to pressure atrophy in other tissues.

\section{Excitability and isometric contractile properties}

Spontaneous contractions of the myooid were observed soon after myooid formation and myooids occasionally contracted with frequencies as high as $2 \mathrm{~Hz}$. Spontaneous contractions were generally from 3 to $15 \mu \mathrm{N}$ in amplitude, but were sometimes as high as $50 \mu \mathrm{N}$. The intensity and frequency of the spontaneous contractions increased immediately after feeding and for several hours thereafter. No difference was observed between the average rheobase values for the adult and neonatal rat myooids. At late times in culture the rheobase values tended to increase (Fig. 5), indicating a slight decrease in excitability. Chronaxie was determined to be linearly dependent on the rheobase $(F<0.05)$ and therefore was not analyzed further.

At all times after myooid formation the specific $\mathrm{P}_{t}$ of myooids was consistently 50\% of specific $\mathrm{P}_{\mathrm{o}}$ (data not shown), and specific $\mathrm{P}_{\mathrm{o}}$ generated by neonatal rat myooids was lower than that generated by adult rat myooids (Fig. 6). With time in culture, the neonatal rat myooid specific $\mathrm{P}_{\mathrm{o}}$ tended to increase, whereas the specific $\mathrm{P}_{\mathrm{o}}$ of adult rat myooids did not change (Fig. 6). When specific $\mathrm{P}_{\mathrm{o}}$ was calculated using the myotube CSA, rather than the total CSA, the difference between the specific $\mathrm{P}_{\mathrm{o}}$ of adult and neonatal rat myooids decreased, and the specific $\mathrm{P}_{\mathrm{o}}$ of neonatal rat myooids was not different from that of adult rat myooids at late time points in culture (Fig. 7). Qualitatively, electrically induced myooid twitches and tetani were very similar to that of control skeletal muscles, although the mean specific $\mathrm{P}_{\mathrm{o}}$ was only $\sim 1 \%$ of control value for adult muscle.

\section{DISCUSSION}

The values for the diameter, rheobase, chronaxie, and specific $\mathrm{P}_{\mathrm{o}}$ of the adult rat myooids agreed with data previously published for adult rat myooids after 31 days in culture, ${ }^{4}$ as well as the specific $\mathrm{P}_{\mathrm{o}}$ from avian organoids. ${ }^{3,4}$ As observed in the earlier study, ${ }^{4}$ the diameter of the adult rat myooids decreased grad-

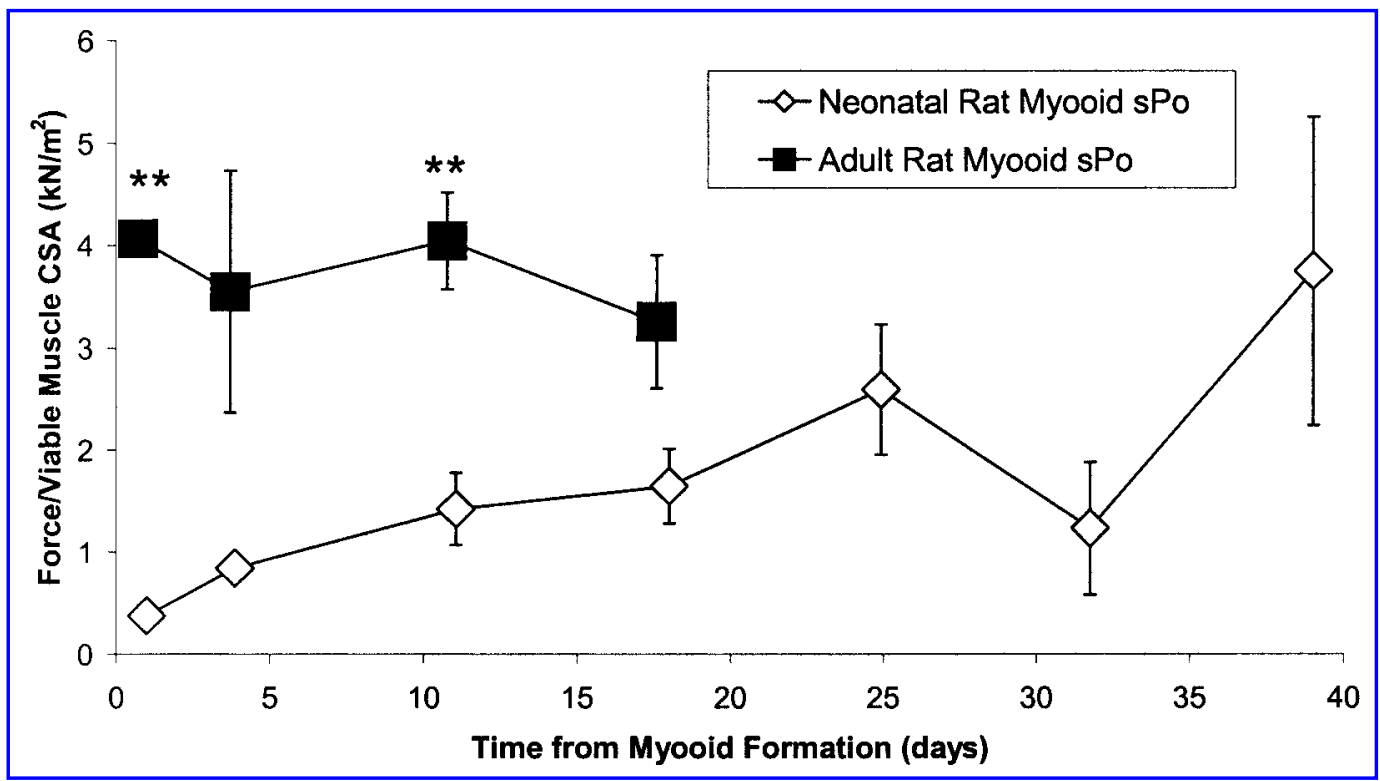

FIG. 7. Specific $\mathrm{P}_{0}$ of adult and neonatal rat myooids calculated using myotube CSA, the total CSA minus the fibroblast CSA. The first datum of each sequence is the average of two myooid specific forces. All other data points for both groups are the mean \pm SEM for four myooid specific forces. Statistically significant differences between the groups: $* p<0.05, * * p<0.01, * * * p<0.001$. 
ually throughout the time the myooids were maintained in culture. In contrast to the $50 \%$ decrease in the diameter of adult myooids from day 4 to 17, the neonatal rat myooids showed a more complex behavior with a twofold increase in diameter between days 25 and 32 followed by a $50 \%$ decrease by day 39 . Immediately following myooid formation, very few cells from either neonatal or adult rats remained on the SYLGARD substrate. Of the cells that remained on the substrate, the greater proliferative capacity of cells from neonatal compared with adult rats ${ }^{12}$ allowed sufficient proliferation of the neonatal cells to form a second confluent monolayer. This secondary monolayer subsequently underwent delamination and incorporation into the existing myooid. The newly incorporated tissue was easily identified in the subsequent cross sections. Further longitudinal studies of myooids engineered from neonatal cells may require the addition of new methods or procedures to prevent the addition of new cells to the myooid from subsequent delaminations of cell monolayers layers. On the other hand, a similar mechanism may be employed by design for building the section thickness of engineered muscle. Ultimately, when the tissue diameter is no longer limited by diffusion distances, such as when capillary networks are engineered into the muscle constructs, with subsequent perfusion, it may be possible to increase the thickness of the engineered construct by sequential layering with monolayers of cells by a mechanism similar to the one observed in this study.

Skeletal muscle constructs developed in culture from embryonic quail, ${ }^{6}$ embryonic chickens with ${ }^{3}$ or without ${ }^{5}$ mechanical stimulation, and adult rats, ${ }^{4}$ each displayed a cross section with fibroblasts and ECM forming an epimysium around the periphery of the muscle construct. In cross sections of muscle constructs from embryonic quail and chicken, septa comprised of fibroblasts and ECM created a perimysium and endomysium. ${ }^{3,5,6}$ In the present study, some myooids were segregated into fascicles by fibroblasts and ECM (Fig. 3), but many were not (Fig. 1). In cases where differences were observed between the cross-sectional structure and composition of myooids and that of other skeletal muscle constructs, the differences may be due to differences between mammalian and avian cells in culture, the culture methods, length of time in culture, or to a sampling error since some of the published observations are based on only a few ${ }^{6,3}$ or even a single construct. ${ }^{5}$

The twofold larger fibroblast CSA of the neonatal compared with the adult rat myooids was unexpected. In earlier work involving myooids engineered exclusively from adult rat cells, ${ }^{4}$ the annulus of fibroblasts was noted, but was only a small percentage of the total CSA. The large difference may have resulted from a methodological difference in cell harvesting from skeletal muscle of neonatal and adult rats. The soleus muscles of the adult rats were pre-incubated before cell culture, whereas the skeletal muscle tissue of the neonatal rats was not. Each method was optimized to harvest the maximum number of satellite cells from each of the tissue sources. The different methods may have had different efficiencies in terms of either harvesting or selecting against fibroblasts. Other possible explanations are a difference in total number of cells, proliferation rate, or proliferation potential between fibroblasts harvested from the neonatal compared with adult rats.

The lower specific $\mathrm{P}_{\mathrm{o}}$ of neonatal rat myooids at all time points in culture resulted in part from the greater percentage of the total CSA consisting of fibroblasts. At early time points after formation, myooids were evaluated and sectioned to permit the specific $\mathrm{P}_{\mathrm{o}}$ to be normalized by myotube CSA. Even with the correction of specific $\mathrm{P}_{\mathrm{o}}$ by normalization by myotube CSA, at early times in culture a deficit remained in the specific $\mathrm{P}_{\mathrm{o}}$ of the neonatal rat compared with the adult rat myooids. The myotubes within the neonatal rat myooids may be at an earlier stage of development than those of the adult rat myooids. The observation that, at late times in culture, the mean specific $\mathrm{P}_{\mathrm{o}}$ of the neonatal rat myooids was not different from that of adult rat myooids when normalized by the myotube CSA supports this premise. Based on these data, our hypothesis that the contractile properties of the neonatal and adult rat myooids are not different was only partially supported, with differences observed only at early time points.

The passive baseline forces generated by fibroblasts are important factors in tissue development, ${ }^{14}$ cell and tissue migration, ${ }^{15}$ and wound closure. ${ }^{13}$ The concept that intrinsic tissue forces are responsible for wound closure has been recognized since Greco-Roman times, ${ }^{13}$ but the possibility that fibroblasts in ECM matrixes may be responsible for the generation of these forces under such circumstances was proposed only recently. ${ }^{7}$ Our data support the hypothesis that myooid baseline force correlates with fibroblast CSA. Based on a simple linear regression model, the specific $\mathrm{P}_{\mathrm{b}}$ of $5.5 \mathrm{kPa}$ for mammalian fibroblasts associated with myooids agrees with the measured stress of avian fibroblasts in a collagen $\operatorname{gel}^{8}$ of $4.5 \mathrm{kPa}$. The measurements of the 
stress generated by avian fibroblasts in their own $\mathrm{ECM}^{9}$ of $3.4 \mathrm{kPa}$ and mammalian wound contraction force ${ }^{10}$ of $3.2 \mathrm{kPa}$ each have recognized systematic errors that underestimate the mechanical stress. Despite these shortcomings, our measurements are in reasonable agreement with the reported values.

Multiple regression on a two-component cylindrical model of the myooids (Appendix) revealed that the fibroblasts exert stresses that are slightly higher than indicated by the simple regression model, perhaps as high as $5.9 \mathrm{kPa}$. The hoop stress generated by the fibroblasts results in a pressure that is exerted upon the core of myoblasts in each myooid of $\sim 1 \mathrm{kPa}$. This explains why the multiple regression calculations indicate that, when relaxed, the core of myotubes exerts a small negative tensile stress; they are being compressed by the ring of fibroblasts, and as a result are being pressed outward toward the ends of the myooid. The continuous pressure exerted by the outer layer of fibroblasts may also explain the inexorable decrease in diameter of myooids with time in culture. The excitability of myooids from neonatal and adult rats are not different, and when normalized for myotube CSA, the differences in specific $\mathrm{P}_{\mathrm{o}}$ between neonatal and adult rat myooids diminishes and eventually disappears with time in culture.

In summary, we conclude that the passive baseline force in engineered skeletal muscle constructs arises largely from the action of fibroblasts. When normalized by cross-sectional area, the passive stress compares favorably with that reported earlier for fibroblasts. ${ }^{8-10}$ Fibroblasts constitute an important cellular component in the early development of self-organizing three-dimensional skeletal muscle constructs in vitro because they provide much of the extracellular matrix that is required to support the cell monolayer during delamination and in the early stages of remodeling. ${ }^{17}$ After formation, however, the large baseline forces that result from the presence of the fibroblasts may contribute to the inexorable reduction in diameter of the constructs with time in culture, and the ultimate tensile failure of the constructs. The action of fibroblasts also appears to place the myotubes under continuous pressure, resulting from the tangential stress exerted by the layer of fibroblasts at the periphery of each cylindrical tissue construct.

\section{ACKNOWLEDGMENTS}

We thank Joseph Chromiak for suggestions on initial methods used to isolate and culture cells from neonatal rats, Cheryl Hasset for assistance with small animal surgeries, and Krystyna Pasyk for histological sections. We also thank Mark Palmer for reviewing the calculations presented in the results. This work was supported by the Contractility Core of the Nathan Shock Center for the Basic Biology of Aging P30 AG13283, and RO1 AG01657.

\section{APPENDIX}

The total baseline passive force was modeled as described in Equation 1:

$$
\mathrm{P}_{\mathrm{b}}=\sigma_{\mathrm{f}} \mathrm{CSA}_{\mathrm{f}}+\sigma_{\mathrm{m}} \mathrm{CSA}_{\mathrm{m}}+\mathrm{P}_{\text {offset }}
$$

The coefficients $\mathrm{P}_{\text {offset }}, \sigma_{\mathrm{m}}$, and $\sigma_{\mathrm{f}}$ are determined by solving the multiple regression problem to find the least squares fit of the data by constructing the equations in matrix form, as $\mathbf{A x}=\mathbf{b}$ :

$$
\left[\begin{array}{ccc}
C S A_{f 1} & C S A_{m 1} & 1 \\
C S A_{f 2} & C S A_{m 2} & 1 \\
\vdots & \vdots & \vdots \\
C S A_{f 20} & C S A_{m 20} & 1
\end{array}\right]\left[\begin{array}{c}
\sigma_{f} \\
\sigma_{m} \\
P_{o f f s e t}
\end{array}\right]=\left[\begin{array}{c}
P_{b 1} \\
P_{b 2} \\
\vdots \\
P_{b 20}
\end{array}\right]
$$

This system of equations is readily solved using MATLAB (The Mathworks Inc., Natick, MA), a commercially-available software package, or may be solved manually by standard row and column operations. Briefly, both sides of the equation are multiplied by the transpose of A to obtain the normal equations:

$$
\mathbf{A}^{\mathbf{T}} \mathbf{A x}=\mathbf{A}^{\mathbf{T}} \mathbf{b}
$$




\section{KOSNIK ET AL.}

The vector of coefficients ( $\mathbf{x})$ is solved for by multiplying both sides of the equation by the inverse of $\mathbf{A}^{\mathbf{T}} \mathbf{A}$ :

$$
\mathbf{x}=\left(\mathbf{A}^{\mathbf{T}} \mathbf{A}\right)^{-1} \mathbf{A}^{\mathbf{T}} \mathbf{b}
$$

The matrix operations of Equations 3 and 4 were carried out within MATLAB using the $\backslash$ symbol in the command: $\mathbf{A l b}$, which automatically returns the least squares solution to the system of equations $\mathbf{A x}=\mathbf{b}$. The resulting values for the coefficients were: $\sigma_{\mathrm{m}}=-0.18 \mathrm{kPa}$, and $\sigma_{\mathrm{f}}=5.9 \mathrm{kPa}$, and $\mathrm{P}_{\text {offset }}=7.7 \times 10^{-8} \mathrm{kN}$.

\section{REFERENCES}

1. Lewis, M.R. Rhythmical contraction of the skeletal muscle tissue observed in tissue cultures. Am. J. Phys. 38, 153, 1915.

2. Lewis, W.H., and Lewis, M.R. Behavior of cross striated muscle in tissue cultures. Am. J. Anat. 22, 169, 1917.

3. Vandenburgh, H.H., Swasdison, S., and Karlisch, P. Computer-aided mechanogenesis of skeletal muscle organs from single cells in vitro. F.A.S.E.B. J. 5, 2860, 1991.

4. Dennis, R.G., and Kosnik, P.E. Excitability and isometric contractile properties of mammalian skeletal muscle constructs engineered in vitro. In Vitro Cell. Dev. Biol. Anim. 36, 327, 2000.

5. Strohman, R.C., Bayne, E., Spector, D., Obinata, T., Micou-Eastwood, J., and Maniotis, A. Myogenesis and histogenesis of skeletal muscle on flexible membranes in vitro. In Vitro Cell. Dev. Biol. 26, 201, 1990.

6. Swasdison, S. and Mayne, R. Formation of highly organized skeletal muscle fibers in vitro. Comparison with muscle development in vivo. J. Cell Sci. 102, 643, 1992.

7. Harris, A.K., Stopak, D., and Wild, P. Fibroblast traction as a mechanism for collagen morphogenesis. Nature 290, $249,1981$.

8. Kolodney, M.S., and Wysolmerski, R.B. Isometric contraction by fibroblasts and endothelial cells in tissue culture: a quantitative study. J. Cell Biol. 117, 73, 1992.

9. James, D.W., and Taylor, J.F. The stress developed by sheets of chick fibroblasts in vitro. Exp. Cell Res. 54, 107, 1969.

10. Higton, D.I.R., and James, D.W. The force of contraction of full-thickness wounds of rabbit skin. Brit. J. Surg. 51, $462,1964$.

11. Cognard, C., Constantin, B., Rivet-Bastide, M., Imbert, N., Besse, C., and Raymond, G. Appearance and evolution of calcium currents and contraction during the early post-fusional stages of rat skeletal muscle cells developing in primary culture. Development 117, 1153, 1993.

12. Decary, S., Mouly, V., Hamida, C.B., Sautet, A., Barbet, J. P., and Butler-Browne, G.S. Replicative potential and telomere length in human skeletal muscle: implications for satellite cell-mediated gene therapy. Hum. Gene Ther. 8, 1429, 1997.

13. Grinnell, F. Fibroblasts, myofibroblasts, and wound contraction. J. Cell. Biol. 124, 401, 1994.

14. Harris, A.K. Physical forces and pattern formation in limb development. In: J.R. Hinchliffe, J.M. Hurle, and D. Summerbell, eds. Developmental Patterning of the Vertebrate Limb. New York: Plenum Press, 1991, pp. 203-210.

15. Harris, A.K., Wild, P., and Stopak, D. Silicone rubber substrata: a new wrinkle in the study of cell locomotion. Science 208, 177, 1980.

16. Shigley, J.E., and Mischke, C.R. Mechanical Engineering Design, 5th ed. New York: McGraw-Hill, 1989, p. 60.

17. Dennis R.G., Kosnik P., Gilbert M.E., and Faulkner, J.A. Excitability and contractility of skeletal muscle engineered from primary cultures and cell lines. Am. J. Physiol. Cell. 280, C288-C295, 2001.

Address reprint requests to:

Robert Dennis, Ph.D.

University of Michigan

300 N. Ingalls, Room 956

Ann Arbor, MI 48109-2007

E-mail: bobden@umich.edu 


\section{This article has been cited by:}

1. Peter J. Yang , Johnna S. Temenoff . 2009. Engineering Orthopedic Tissue InterfacesEngineering Orthopedic Tissue Interfaces. Tissue Engineering Part B: Reviews 15:2, 127-141. [Abstract] [Full Text] [PDF] [PDF Plus]

2. Kristel J.M. Boonen , Mark J. Post . 2008. The Muscle Stem Cell Niche: Regulation of Satellite Cells During RegenerationThe Muscle Stem Cell Niche: Regulation of Satellite Cells During Regeneration. Tissue Engineering Part B: Reviews 14:4, 419-431. [Abstract] [PDF] [PDF Plus]

3. Rahul G. Thakar, Matthew G. Chown, Anuj Patel, Lily Peng, Sanjay Kumar, Tejal A. Desai. 2008. Contractility-Dependent Modulation of Cell Proliferation and Adhesion by Microscale Topographical Cues. Small 4:9, 1416-1424. [CrossRef]

4. Ravi Birla, Vikas Dhawan, Yen-Chih Huang, Ian Lytle, Khajohn Tiranathanagul, David Brown. 2008. Force Characteristics of In Vivo Tissue-engineered Myocardial Constructs Using Varying Cell Seeding Densities. Artificial Organs 32:9, 684-691. [CrossRef]

5. R. K. Birla, Y. C. Huang, R. G. Dennis. 2008. Effect of streptomycin on the active force of bioengineered heart muscle in response to controlled stretch. In Vitro Cellular \& Developmental Biology - Animal 44:7, 253-260. [CrossRef]

6. Luda Khait, Louise Hecker, Desmond Radnoti, Ravi K. Birla. 2008. Micro-Perfusion for Cardiac Tissue Engineering: Development of a Bench-Top System for the Culture of Primary Cardiac Cells. Annals of Biomedical Engineering 36:5, 713-725. [CrossRef]

7. Vikas Dhawan, Ian F. Lytle, Douglas E. Dow, Yen-Chih Huang, David L. Brown . 2007. Neurotization Improves Contractile Forces of Tissue-Engineered Skeletal MuscleNeurotization Improves Contractile Forces of Tissue-Engineered Skeletal Muscle. Tissue Engineering 13:11, 2813-2821. [Abstract] [PDF] [PDF Plus]

8. Robert G. Dennis , Douglas E. Dow . 2007. Excitability of Skeletal Muscle during Development, Denervation, and Tissue CultureExcitability of Skeletal Muscle during Development, Denervation, and Tissue Culture. Tissue Engineering 13:10, 2395-2404. [Abstract] [PDF] [PDF Plus]

9. R.K. Birla , Y.C. Huang , R.G. Dennis . 2007. Development of a Novel Bioreactor for the Mechanical Loading of Tissue-Engineered Heart MuscleDevelopment of a Novel Bioreactor for the Mechanical Loading of Tissue-Engineered Heart Muscle. Tissue Engineering 13:9, 2239-2248. [Abstract] [PDF] [PDF Plus]

10. Michelle Hairfield-Stein , Christopher England, Hyun J. Paek , Kerry B. Gilbraith , Robert Dennis , Eugene Boland , Paul Kosnik . 2007. Development of Self-Assembled, Tissue-Engineered Ligament from Bone Marrow Stromal CellsDevelopment of Self-Assembled, Tissue-Engineered Ligament from Bone Marrow Stromal Cells. Tissue Engineering 13:4, 703-710. [Abstract] [PDF] [PDF Plus]

11. Louise Hecker, Ravi K Birla. 2007. Engineering the heart piece by piece: state of the art in cardiac tissue engineering. Regenerative Medicine 2:2, 125-144. [CrossRef]

12. Michelle Hairfield-Stein, Christopher England, Hyun J. Paek, Kerry B. Gilbraith, Robert Dennis, Eugene Boland, Paul Kosnik. 2007. Development of Self-Assembled, Tissue-Engineered Ligament from Bone Marrow Stromal Cells. Tissue Engineering, ahead of print070116053433001. [CrossRef]

13. Michelle Hairfield-Stein, Christopher England, Hyun J. Paek, Kerry B. Gilbraith, Robert Dennis, Eugene Boland, Paul Kosnik. 2007. Development of Self-Assembled, Tissue-Engineered 
Ligament from Bone Marrow Stromal Cells. Tissue Engineering, ahead of print070108073551001. [CrossRef]

14. Lisa M. Larkin, Sarah Calve, Tatiana Y. Kostrominova, Ellen M. Arruda. 2006. Structure and Functional Evaluation of Tendon?Skeletal Muscle Constructs Engineered in Vitro. Tissue Engineering, ahead of print061012064037003. [CrossRef]

15. Lisa M. Larkin , Sarah Calve, Tatiana Y. Kostrominova, Ellen M. Arruda . 2006. Structure and Functional Evaluation of Tendon-Skeletal Muscle Constructs Engineered in VitroStructure and Functional Evaluation of Tendon-Skeletal Muscle Constructs Engineered in Vitro. Tissue Engineering 12:11, 3149-3158. [Abstract] [PDF] [PDF Plus]

16. Justus P. Beier, Jens Stern-Straeter, Vanni T. Foerster, Ulrich Kneser, G Bjoern Stark, Alexander D. Bach. 2006. Tissue Engineering of Injectable Muscle: Three-Dimensional Myoblast-Fibrin Injection in the Syngeneic Rat Animal Model. Plastic and Reconstructive Surgery 118:5, 1113-1121. [CrossRef]

17. A. D. Bach, A. Arkudas, J. Tjiawi, E. Polykandriotis, U. Kneser, R. E. Horch, J. P. Beier. 2006. A new approach to tissue engineering of vascularized skeletal muscle. Journal of Cellular and Molecular Medicine 10:3, 716-726. [CrossRef]

18. David L. Brown, D Keith Bishop, Sherri Y. Wood, Paul S. Cederna. 2006. Short-Term Anti-CD40 Ligand Costimulatory Blockade Induces Tolerance to Peripheral Nerve Allografts, Resulting in Improved Skeletal Muscle Function. Plastic and Reconstructive Surgery 117:7, 2250-2258. [CrossRef]

19. Gregory H. Borschel, Douglas E. Dow, Robert G. Dennis, David L. Brown. 2006. Tissue-Engineered Axially Vascularized Contractile Skeletal Muscle. Plastic and Reconstructive Surgery 117:7, 2235-2242. [CrossRef]

20. LISA M. LARKIN, JACK H. VAN DER MEULEN, ROBERT G. DENNIS, JEFFREY B. KENNEDY. 2006. FUNCTIONAL EVALUATION OF NERVE-SKELETAL MUSCLE CONSTRUCTS ENGINEERED IN VITRO. In Vitro Cellular \& Developmental Biology - Animal 42:3, 75. [CrossRef]

21. A. D. Bach, J. P. Beier, J. Stern-Staeter, R. E. Horch. 2004. Skeletal muscle tissue engineering. Journal of Cellular and Molecular Medicine 8:4, 413-422. [CrossRef]

22. Gregory H. Borschel, Robert G. Dennis, William M. Kuzon. 2004. Contractile Skeletal Muscle Tissue-Engineered on an Acellular Scaffold. Plastic and Reconstructive Surgery 113:2, 595-602. [CrossRef]

23. Joanne Garvin , Jie Qi , Melissa Maloney, Albert J. Banes . 2003. Novel System for Engineering Bioartificial Tendons and Application of Mechanical LoadNovel System for Engineering Bioartificial Tendons and Application of Mechanical Load. Tissue Engineering 9:5, 967-979. [Abstract] [PDF] [PDF Plus]

24. ERIN L. BAKER, ROBERT G. DENNIS, LISA M. LARKIN. 2003. GLUCOSE TRANSPORTER CONTENT AND GLUCOSE UPTAKE IN SKELETAL MUSCLE CONSTRUCTS ENGINEERED IN VITRO. In Vitro Cellular \& Developmental Biology - Animal 39:10, 434. [CrossRef] 